\title{
Laboratory diagnosis of COVID-19 in secondary care hospitals in India: will standalone serology suffice?
}

\author{
Sara Chandy ${ }^{1} \cdot$ Sundaram Balasubramanian ${ }^{2} \cdot$ Anand Manoharan $^{1}(\mathbb{C}$
}

Received: 19 August 2020 / Accepted: 24 November 2020 / Published online: 23 January 2021

(c) Children's Hospital, Zhejiang University School of Medicine 2021

RT-PCR is the accepted gold standard for diagnosis of COVID-19. The specificity of RT-PCR is very good, but its sensitivity largely depends on the type of sample collected, timing of collection, clinical sampling protocol, and nucleic acid detection technology employed. The detection rate of RT-PCR compared with chest CT is $<70 \%$ during the acute phase ( $0-7$ days post onset (DPO) and is only $50 \%$ by the second week of illness. Positivity from sputum and nasal swabs is $74.4-88.9 \%$ and $53.6-73.3 \%$, respectively. RT-PCR is expensive and requires specialized laboratory equipment and trained personnel [1]. Reliable antigen detection tests for quick detection of COVID-19 patients remain unavailable in India.

While most paediatric patients with COVID-19 have had an uneventful clinical course and about a quarter of them are reportedly asymptomatic, they may play a significant role in the community transmission of the virus. IgM and IgG antibodies against SARS-CoV-2 have been detected as early as 4 DPO. Serological testing can be considered as an alternative to RT-PCR for early diagnosis and is recommended for children with multisystem inflammatory syndrome (MIS-C). Serological testing has a faster turn-around time, higher throughput, less workload and can be used in low resource settings [2].

In India, secondary care centers cater to the semi-urban and rural populations including children. These centers usually perform basic microbiological testing including serology. During a pandemic of the magnitude of COVID-19, these centers may benefit immensely from availability of affordable and accurate serological tests. We review the diagnostic value of incorporating total antibody (IgM, IgA and $\operatorname{IgG}$ ), $\operatorname{IgM}$,

Anand Manoharan

anandmanoharan@ctmrf.org

1 Molecular Laboratory, The CHILDS Trust Medical Research Foundation, Chennai, Tamil Nadu 600034, India

2 Department of Pediatrics, Kanchi Kamakoti CHILDS Trust Hospital, Chennai, Tamil Nadu 600034, India and $\mathrm{IgG}$ assays in routine COVID-19 testing in laboratories where molecular testing may be unaffordable [2].

The 30-Kb RNA of SARS-CoV-2, the etiological agent of COVID-19, has four major structural proteins encoded by one-third of the genome. These include the phosphorylated nucleocapsid protein $(\mathrm{N})$ at the core, a small membrane glycoprotein (M), club-shaped peplomer glycoproteins (S) extending from the virus surface as spikes, and the envelope (E) protein. $\mathrm{N}$ and $\mathrm{S}$ are immunodominant proteins, largely being employed in serological assays to detect antibodies to SARS-CoV-2 [3].

The $50-\mathrm{kDa} \mathrm{N}$ protein is produced copiously in infected cells during early infection. Lack of glycosylation makes it an easy antigen (both as complete and partial protein) to clone and to express in bacterial vectors. The homology of the amino acid (aa) sequence of SARS-CoV-2 N protein to several other coronaviruses, including MERS-CoV, HKU1, OC43, NL63, 229E and SARS-CoV, is 46.1\%, $27.6 \%, 26.5 \%, 20.0 \%, 19.1 \%$ and $90.5 \%$, respectively $[3,4]$. The spike S protein of SARS-CoV-2 has only about $80 \%$ sequence identity with known coronaviruses.

Full-length nucleocapsid (N) genes of SARS-CoV-2 (expressed in E.coli) that are employed in an indirect ELISA could detect IgM, IgA, and IgG at 1 DPO. IgA and IgM positivity in acute samples (1-7 DPO) was $85.4 \%$ and $92.7 \%$, respectively. The median time of detection using IgM and IgA was 5 DPO (IQR: 3-6 DPO). After 5.5 DPO, IgM had greater sensitivity than RT-PCR. When used in combination, sensitivity improved significantly from $51.9 \%$ (RTPCR alone) to $98.6 \%$ (RT-PCR + IgM ELISA) [4]. However, recombinant nucleocapsid $(\mathrm{rN})$ protein in mu-capture ELISA format had lower sensitivity $(<60 \%)$ during the acute stage of the illness (0-10 DPO) [5].

Full and partial glycosylated $\mathrm{S}$ protein expressed in mammalian cells and in insect vectors using baculovirus have been used in serological testing. Anti-S antibodies arise later than anti-N antibodies; hence, the utility of S protein in acute diagnosis is doubtful. The receptor binding domain (RBD) 
of $\mathrm{S}$ protein interacts with angiotensin-converting enzyme 2 (ACE2) and mediates attachment to host cells. A total antibody $(\operatorname{Ig} \mathrm{A}+\operatorname{IgM}+\operatorname{IgG})$ ELISA with $\mathrm{rRBD}$ had a sensitivity of $64.1 \%$ in acute samples (0-7 DPO) compared with IgM and IgG (both at 33.3\%, $P<0.001$ ) [6]. However, the positivity rate employing rRBD in a $\mu$-chain IgM capture ELISA format during the early phase of illness was only $38.3 \%$ compared with $66.7 \%$ of RT-PCR. Even though SARS-CoV-2 anti-S IgM antibodies were detectable from day 4 onwards, less than $60 \%$ of samples collected during the early stage of the illness (0-10 DPO) showed IgM positivity. Severe cases of COVID-19 documented a vigorous IgG and IgM response after 7-10 DPO [7].

Tandem testing with recombinant SARS-CoV-2 N protein (residue 1-419) and with partial S protein (residue 16-685) for $\mathrm{IgM}$ and $\mathrm{IgG}$ detection has produced reasonable results. In acute samples, seropositivity of anti-S IgM + anti-S IgG/anti-N $\mathrm{IgG}+$ anti-S IgG was $66.7 \%$, whereas that of anti-N IgM+ anti$\mathrm{S}$ IgM+anti-N IgG+ anti-S IgG was higher (75\%) [8].

The genetic relatedness of the $\mathrm{N}$ and $\mathrm{S}$ immunogenic structural proteins of closely related coronaviruses (NL63, 229E, OC43, HKU1, SARS-CoV, MERS-CoV) can elicit crossreactive antibodies in the host. The structural $\mathrm{N}$ protein is largely conserved among coronaviruses; a strong cross-reactivity between SARS-CoV positive human plasma and SARSCoV-2 rNP has been reported [4]. However, the immunodominant regions of the $\mathrm{S}$ protein vary in their relatedness to other beta coronaviruses. Therefore, $\mathrm{N}$-based assays show a greater degree of cross-reactivity than $\mathrm{S}$ antigen-based assays. Most of the serological studies discussed here to study cross-reactivity of the assay have employed control sera from healthy individuals collected before the outbreak of COVID-19.

The ongoing COVID-19 is the third coronavirus outbreak of this century after SARS in 2003 and MERS in 2012. A pressing need exists for an alternative to RT-PCR in view of its limitations. However, none of the reported serological systems, including lateral flow assays (LFA), has demonstrated good accuracy indices for diagnosis during the acute stage.

In summary, the use of serodiagnostic assays for coronaviruses in the acute stage of illness is limited. There are multiple reasons for this. Importantly, the detection of IgM antibodies, the marker of acute infection, is often delayed up to 10-12 DPO. Furthermore, there is significant crossreactivity between the circulating coronaviruses [9].

Nucleocapsid antibodies appear earlier than spike antibodies. Cross-reactivity between the largely conserved $\mathrm{N}$ protein of closely related coronaviruses may give false positives. RBD, which has been used in many laboratories, represents a small part of the $S$ protein ( 237 aa versus 273 aa of $\mathrm{S}$ protein), and an anti-RBD IgM IgG response may not represent the whole array of host antibody responses to $S$ protein [10]. It needs to be validated with a larger sample set before routine use. Choice of the antigen and the ELISA format (indirect or mu-capture) has a bearing on accuracy indices. Whether a cocktail of $\mathrm{N}$ and $\mathrm{S}$ antigens may improve sensitivity during the acute phase is worth investigating. Total antibody detection is superior in sensitivity to IgM and IgG detection and should be explored further.

In light of these results, the utility of serological assays for early diagnosis of COVID -19 during the acute phase of the illness remains to be demonstrated.

Author contributions All authors contributed to the preparation of this viewpoint manuscript, and the final version was approved by all.

Funding None.

\section{Compliance with ethical standards}

Ethical approval Not applicable for this viewpoint.

Conflict of interest The authors declare that they have no competing interest.

\section{References}

1. Fang Y, Zhang H, Xie J, Lin M, Ying L, Pang P, et al. Sensitivity of chest CT for COVID-19: comparison to RT-PCR. Radiology. 2020;296:E115-7.

2. Feldstein LR, Rose EB, Horwitz SM, Collins JP, Newhams MM, Son MBF, et al. Multisystem inflammatory syndrome in US children and adolescents. N Engl J Med. 2020;383:334-46.

3. Lu R, Zhao X, Li J, Niu P, Yang B, Wu H, et al. Genomic characterisation and epidemiology of 2019 novel coronavirus: implications for virus origins and receptor binding. Lancet. 2020;395:565-74.

4. Guo L, Ren L, Yang S, Xiao M, Chang D, Yang F, et al. Profiling early humoral response to diagnose novel coronavirus disease (COVID-19). Clin Infect Dis. 2020;71:778-85.

5. Liu W, Liu L, Kou G, Zheng Y, Ding Y, Ni W, et al. Evaluation of nucleocapsid and spike protein-based enzyme-linked immunosorbent assays for detecting antibodies against SARS-CoV-2. J Clin Microbiol. 2020;58:e0461-520.

6. Lou B, Li TD, Zheng SF, Su YY, Li ZY, Liu W, et al. Serology characteristics of SARS-CoV-2 infection after exposure and postsymptom onset. Eur Respir J. 2020;56:2000763.

7. Zhao J, Yuan Q, Wang H, Liu W, Liao X, Su Y, et al. Antibody responses to SARS-CoV-2 in patients of novel coronavirus disease 2019.Clin Infect Dis. 2020;71:2027-34.

8. Sun B, Feng Y, Mo X, Zheng P, Wang Q, Li P, et al. Kinetics of SARS-CoV-2 specific IgM and IgG responses in COVID-19 patients. Emerg Microbes Infect. 2020;9:940-8.

9. Burbelo PD, Riedo FX, Morishima C, Rawlings S, Smith D, Das S, et al. Sensitivity in detection of antibodies to nucleocapsid and spike proteins of severe acute respiratory syndrome coronavirus 2 in patients with coronavirus disease 2019. J Infect Dis. 2020;222:206-13.

10. Meyer B, Drosten C, Müller MA. Serological assays for emerging coronaviruses: challenges and pitfalls. Virus Res. 2014;194:175-83.

Publisher's Note Springer Nature remains neutral with regard to jurisdictional claims in published maps and institutional affiliations. 